\title{
EVERY SEPARABLE BANACH SPACE IS ISOMETRIC TO A SPACE OF CONTINUOUS NOWHERE DIFFERENTIABLE FUNCTIONS
}

\author{
L. RODRÍGUEZ-PIAZZA
}

(Communicated by Dale Alspach)

\begin{abstract}
We prove the result stated in the title; that is, every separable Banach space is linearly isometric to a closed subspace $E$ of the space of continuous functions on $[0,1]$, such that every nonzero function in $E$ is nowhere differentiable.
\end{abstract}

\section{INTRODUCTION}

A theorem of Banach and Mazur [B2] states that every separable Banach space $X$ is isometrically embeddable in $\mathscr{C}([0,1])$. One can ask whether we can require more properties of the functions in the image of the embedding; or, when these properties establish restrictions on the Banach space $X$.

In this direction, it has been proved that a closed subspace $E$ of $\mathscr{C}([0,1])$ must be finite-dimensional if every function in $E$ has bounded variation [LM], or if every function in $E$ is differentiable at every point of [0,1] [G1]. If $E$ is infinite-dimensional and every function in $E$ has a derivative at every point of $(0,1]$, then $E$ must contain an isomorphic copy of $c_{0}$ [G1]; in fact, $E$ must be isomorphic to a subspace of $c_{0}$. Moreover, if we want to embed $\ell_{1}$ in $\mathscr{C}([0,1])$, we will always find a function in the image of the embedding which is nondifferentiable at every point of a perfect subset of $[0,1]$ [PT]. Our aim is to prove that nowhere differentiability imposes no restriction for embedding any separable Banach space.

Weierstrass was the first one to give an example of a continuous nowhere differentiable function [W]. Actually, almost all functions in $\mathscr{C}([0,1])$, in the sense of Baire's category, are nowhere differentiable, as was proved by Banach [B1]. In [G2] an infinite-dimensional subspace of such functions (of course, but the zero function) is constructed using trigonometric sums. In [FGK] the authors use Van der Waerden's functions for giving such a closed subspace of $\mathscr{C}([0,1])$ isomorphic to $\ell_{1}$.

Received by the editors October 15, 1993.

1991 Mathematics Subject Classification. Primary 46B04, 26 A27.

Key words and phrases. Continuous nondifferentiable functions, Banach spaces, isometric linear embeddings.

The author was partially supported by DGICYT grant PB90-893. 
We are going to improve these two last results: we will prove that every separable Banach space can be isometrically embedded in $\mathscr{C}([0,1])$ using only the zero function and nowhere differentiable functions. Let $\Delta$ be the Cantor set $\left(\Delta=\{0,1\}^{\mathbb{N}}\right)$; it is well known that every separable Banach space is isometric to a subspace of $\mathscr{C}(\Delta)$, so we only have to consider this space to obtain the announced result for every separable space. This case will be stated in the following theorem; we devote the rest of the paper to its proof.

Theorem 1. There exist a closed subset $K$ of $[0,1]$, homeomorphic to the Cantor set $\Delta$, and a linear operator $S: \mathscr{C}(K) \rightarrow \mathscr{C}([0,1])$ such that, for every $f \in$ $\mathscr{C}(K) \backslash\{0\}$, we have:

(i) $S f(t)=f(t)$ for every $t \in K$; that is, $S f$ is a continuous extension of $f$ to the whole interval.

(ii) $\|S f\|_{\infty}=\|f\|_{\infty}, S$ is an isometry.

(iii) $S f$ is nowhere differentiable in $[0,1]$.

In order to prove this theorem, after giving the description of $K$, we will construct a first operator $T: \mathscr{C}(K) \rightarrow \mathscr{C}([0,1])$ which satisfies (i) and (ii) in the theorem, but such that, for every $f$ in $\mathscr{C}(K), T f$ behaves well, that is, we can control both the infinity norm in certain subsets, and certain increments. The operator $S$ will be a perturbation of $T$.

\section{DESCRIPTION OF $K$ AND $T$}

Let $\left(m_{n}\right)_{n \geq 0}$ be an increasing sequence of natural numbers such that $m_{0}=1$ and that, for every $n=1,2,3, \ldots$, we have

$$
\frac{m_{n}}{m_{n-1}} \text { is an integer divisible by } 4, \quad \frac{m_{n}}{m_{n-1}} \geq \frac{10 \cdot 2^{n+2}}{1 / 6} .
$$

This implies that, for every $n \geq 1$, we have

$$
\sum_{k=1}^{n-1} \frac{2^{n+1} m_{k}}{2^{k} m_{n}}<1 / 6
$$

For every $n=0,1,2, \ldots$ we will choose $2^{n}$ pairwise disjoint closed intervals $\left\{I_{n, j}\right\}_{j=1}^{2^{n}}$ in $[0,1]$ such that, if $I_{n, j}=\left[a_{n, j}, b_{n, j}\right]$, then

$$
m_{n} a_{n, j} \text { is an integer divisible by } 4 \text {, and } b_{n, j}=a_{n, j}+\frac{1}{m_{n}} \text {. }
$$

We will also require that

$$
\begin{gathered}
I_{n+1,2 j-1} \cup I_{n+1,2 j} \subset \text { the interior of } I_{n, j}, \\
n=0,1,2, \ldots, \quad j=1,2, \ldots, 2^{n} .
\end{gathered}
$$

In this situation, it is clear that, if

$$
K_{n}=I_{n, 1} \cup I_{n, 2} \cup \ldots \cup I_{n, 2^{n}},
$$

then $K=\bigcap_{n>1} K_{n}$ is homeomorphic to the Cantor set $\Delta$.

Let us define the intervals $I_{n, j}$. There is no other possible selection for the case $n=0, I_{0,1}$ must be $[0,1]$. Suppose that for certain $n$ we have defined $I_{n, j}$. Divide this interval into five intervals of equal length $1 / 5 m_{n}$; we will 
choose the interval $I_{n+1,2 j-1}$ inside the second one, and $I_{n+1,2 j}$ inside the fourth one, this will assure us that

$$
\begin{gathered}
a_{n+1,2 j-1}-a_{n, j} \geq \frac{1}{5 m_{n}}, \quad a_{n+1,2 j}-b_{n+1,2 j-1} \geq \frac{1}{5 m_{n}}, \\
\text { and } b_{n, j}-b_{n+1,2 j} \geq \frac{1}{5 m_{n}} .
\end{gathered}
$$

In order to see that we can make this choice with the extremes of the intervals still satisfying (3), take into account that in any of the five subintervals into which we have divided $I_{n, j}$ there are at least $m_{n+1} / 5 m_{n}>5$ consecutive points of the form $x / m_{n+1}$ with $x$ an integer, and we can take some $x$ divisible by 4 such that $x / m_{n+1}$ and $(x+1) / m_{n+1}$ belong to the same subinterval. This completes the construction of $K$.

Now we are going to see that we can extend every continuous function on $K$ to a continuous function on $[0,1]$ controlling certain increments and the infinity norm out of $K_{n}$. This extension will be a linear operator; actually we are going to prove the following

Lemma 2. There exists a linear operator $T: \mathscr{C}(K) \rightarrow \mathscr{C}([0,1])$ satisfying, for every $f \in \mathscr{C}(K)$ :

(a) $T f(t)=f(t)$ for every $t \in K$ ( $T f$ is an extension of $f)$.

(b) $|T f(t)| \leq\left(1-2^{-n}\right)\|f\|_{\infty}$ for every $t \notin K_{n}$.

(c) For every $k=0,1,2, \ldots, m_{n}-1$ we have

$$
\left|T f\left(\frac{k}{m_{n}}\right)-T f\left(\frac{k+1}{m_{n}}\right)\right| \leq \frac{1 / 6}{2^{n+2}}\|f\|_{\infty} .
$$

Properties (a) and (b) imply that $T$ is an isometry.

Proof of Lemma 2. For every $n \geq 0$ and every $j$ pick a point $x_{n, j}$ in $I_{n, j} \cap K$. Let $f$ be in $\mathscr{C}(K)$. We define $T f(t)=f(t)$ for every $t$ in $K$; this satisfies (a). For every $n \geq 0$ and every $j$, we define

$$
T f\left(a_{n, j}\right)=T f\left(b_{n, j}\right)=\left(1-2^{-n}\right) f\left(x_{n, j}\right) .
$$

Extend $T f$ affinely to the interior of every interval in which is not defined.

$T f$ is a continuous function on $[0,1]$ : every point outside $K$ has a neighbourhood where $T f$ is piecewise affine and continuous; for the continuity of $T f$ in $K$ it is enough to remark that the oscillation of $T f$ in each $I_{n, j}$ is less than the oscillation of $f$ in $K \cap I_{n, j}$ plus $2^{-n}\|f\|_{\infty}$. It is easy to see that $T$ is a linear operator.

Take $n \geq 1$ and $t \in K_{n-1} \backslash K_{n}$. Then there will exist a $j$ such that $t$ belongs to one of the three intervals

$$
\left[a_{n-1, j}, a_{n, 2 j-1}\right],\left[b_{n, 2 j-1}, a_{n, 2 j}\right] \text {, or }\left[b_{n, 2 j}, b_{n-1, j}\right] \text {. }
$$

Since $T f$ is affine in each one of these intervals, $|T f(t)|$ is bounded by the maximum of $|T f|$ on the extremes, which is, by the construction of $T f$, less than or equal to $\left(1-2^{-n}\right)\|f\|_{\infty}$, proving (b).

In order to prove (c), remark that if $I$ is one of the intervals in (5), and $x, y \in I$, we have by (4),

$$
|T f(x)-T f(y)| \leq \frac{\text { oscillation of } T f \text { in } I}{\text { length of } I}|x-y| \leq \frac{2\|f\|_{\infty}}{1 / 5 m_{n-1}}|x-y|
$$


If $k / m_{n}$ is one of the points $a_{n, j}$, then $(k+1) / m_{n}=b_{n, j}$ and $T f\left(a_{n, j}\right)=$ $T f\left(b_{n, j}\right)$ by the construction of $T f$, so we have (c); if not, the interval $\left(k / m_{n},(k+1) / m_{n}\right)$ does not meet $K_{n}$ and we can use (6) to obtain, for certain $\nu \leq n$,

$$
\left|T f\left(\frac{k}{m_{n}}\right)-T f\left(\frac{k+1}{m_{n}}\right)\right| \leq 10\|f\|_{\infty} m_{\nu-1} \frac{1}{m_{n}} \leq \frac{10 m_{n-1}}{m_{n}}\|f\|_{\infty} .
$$

This inequality, using (1), implies (c).

\section{Proof of Theorem 1}

To prove the theorem we are going to define $S$ as a perturbation of the operator $T$ in Lemma 2. We will need the following continuous function $g$ defined on the real line: $g$ has period 4 and its values on $[0,4]$ are given by

$$
g(t)= \begin{cases}0, & \text { if } t \in[0,1], \\ t-1, & \text { if } t \in[1,2], \\ 1, & \text { if } t \in[2,3] \\ 4-t, & \text { if } t \in[3,4]\end{cases}
$$

It is easy to see that $g(x)=0$ for every integer $x$ divisible by 4 , that

$$
|g(x)-g(x+2)|=1 \quad \text { for every integer } x,
$$

and that

$$
|g(x)-g(y)| \leq|x-y| \text { for every } x, y \in \mathbb{R} .
$$

Proof of Theorem 1. Take a dense sequence $\left(s_{n}\right)$ in $K$ and, for every $t \in[0,1]$ and every $f \in \mathscr{C}(K)$, define

$$
S f(t)=T f(t)+\sum_{n=1}^{\infty} \frac{f\left(s_{n}\right)}{2^{n+1}} g\left(m_{n} t\right) .
$$

It is obvious that $S: \mathscr{C}(K) \rightarrow \mathscr{C}([0,1])$ is a bounded linear operator. We are going to see that $S$ satisfies the statements of Theorem 1 .

In order to check (i) and (ii), note that if $t \in I_{n, j}=\left[a_{n, j}, b_{n, j}\right]$, then, by (3), $g\left(m_{n} t\right)=0$, thanks to the definition of $g$ on $[0,1]$. So, if $t \in K_{n}$, $g\left(m_{n} t\right)=0$. This and (a) in Lemma 2 prove (i). This also allows us to prove (ii), since, if $t \in K_{n-1} \backslash K_{n}$, we have

$$
\left|\sum_{\nu=1}^{\infty} \frac{f\left(s_{\nu}\right)}{2^{\nu+1}} g\left(m_{\nu} t\right)\right|=\left|\sum_{\nu=n}^{\infty} \frac{f\left(s_{\nu}\right)}{2^{\nu+1}} g\left(m_{\nu} t\right)\right| \leq \sum_{\nu=n}^{\infty} \frac{\|f\|_{\infty}}{2^{\nu+1}}=\frac{\|f\|_{\infty}}{2^{n}}
$$

and by (b) in Lemma 2, (ii) follows.

To finish the proof, suppose that $f \in \mathscr{C}(K) \backslash\{0\}$, and let $D_{f}$ be the infinite subset of integers

$$
D_{f}=\left\{n \in \mathbb{N}:\left|f\left(s_{n}\right)\right|>(1-1 / 6)\|f\|_{\infty}\right\} .
$$

We will prove the following 
Claim. If $n \in D_{f}$ and $k \in\left\{0,1,2, \ldots, m_{n}-2\right\}$, we have

$$
\left|S f\left(\frac{k}{m_{n}}\right)-S f\left(\frac{k+2}{m_{n}}\right)\right| \geq \frac{1 / 2}{2^{n+1}}\|f\|_{\infty} .
$$

(iii) follows from the claim: were $S f$ differentiable at $x_{0}$, there would exist a constant $M$ such that

$$
\left|S f(x)-S f\left(x_{0}\right)\right| \leq M\left|x-x_{0}\right| \text {, for every } x \in[0,1] .
$$

So, if $a \leq x_{0} \leq b$, we would also have $|S f(a)-S f(b)| \leq M(b-a)$. This would be a contradiction with the claim, since we can choose $a=k / m_{n}$ and $b=(k+2) / m_{n}$ for a suitable $k$, and the sequence $m_{n} / 2^{n+2}$ tends to infinity thanks to (1).

Proof of the claim. Since $m_{l} / m_{n}$ is an integer divisible by 4 , for every $l>n$, we have

$$
g\left(m_{l} \frac{k}{m_{n}}\right)=g\left(m_{l} \frac{k+2}{m_{n}}\right)=0 .
$$

By (c) in Lemma 2, we have

$$
\left|T f\left(\frac{k}{m_{n}}\right)-T f\left(\frac{k+2}{m_{n}}\right)\right| \leq \frac{1 / 6}{2^{n+1}}\|f\|_{\infty} .
$$

From (8) and (2) we obtain

$$
\begin{aligned}
& \sum_{\nu=1}^{n-1} \frac{\left|f\left(s_{\nu}\right)\right|}{2^{\nu+1}}\left|g\left(m_{\nu} \frac{k}{m_{n}}\right)-g\left(m_{\nu} \frac{k+2}{m_{n}}\right)\right| \\
& \quad \leq \sum_{\nu=1}^{n-1} \frac{m_{\nu}}{2^{\nu+1}} \frac{2}{m_{n}}\|f\|_{\infty} \leq \frac{1 / 6}{2^{n+1}}\|f\|_{\infty} .
\end{aligned}
$$

From (7), (9), (10), (11) and the definition of $D_{f}$, we get

$$
\begin{aligned}
& \left|S f\left(\frac{k}{m_{n}}\right)-S f\left(\frac{k+2}{m_{n}}\right)\right| \\
& \quad \geq \frac{\left|f\left(s_{n}\right)\right||g(k)-g(k+2)|}{2^{n+1}}-\frac{1 / 6}{2^{n+1}}\|f\|_{\infty}-\frac{1 / 6}{2^{n+1}}\|f\|_{\infty} \geq \frac{1 / 2}{2^{n+1}}\|f\|_{\infty}
\end{aligned}
$$

as claimed.

\section{ACKNOWLEDGEMENTS}

I would like to thank Professor V. I. Gurarii for proposing the problem treated here and for fruitful discussions during the preparation of this paper.

\section{REFERENCES}

[B1] S. Banach, Uber die Baire'sche kategorie gewisser Funktionenmengen, Studia Math. 3 (1931), 174-180.

[B2] _ Théorie des opérations linéaires, PWN, Warsaw, 1932.

[FGK] V. P. Fonf, V. I. Gurarii, and M. J. Kadec, On certain subspace of $\mathscr{C}$, preprint.

[G1] V. I. Gurarii, Subspaces of differentiable functions in the space of continuous functions, Teor. Funktsii Funktsional. Anal. i Prilozhen. 4 (1967), 161-121. (Russian) 
[G2] Linear spaces composed of everywhere nondifferentiable functions, C. R. Acad. Bulgare Sci. 44 (1991), 13-16. (Russian)

[LM] B. Levine and D. Milman, On linear sets in space $\mathscr{C}$ consisting of functions of bounded variation, Comm. Inst. Sci. Math. Méc. Univ. Kharkoff 16 (1940), 102-105. (Russian)

[PT] P. P. Petrushev and S. L. Troyanski, On the Banach-Mazur theorem on the universallity of $\mathscr{C}[0,1]$, C. R. Acad. Bulgare Sci. 37 (1984), 283-285. (Russian)

[W] K. Weierstrass, Mathematische Werke. II, Mayer and Müller, Berlin, 1895, pp. 71-74.

Facilltad de Matemáticas, Aptdo. 1160, Universidad de Sevilla, 41080 Sevilla, SPain

E-mail address: piazzaC cica.es 